\title{
Body Integrity Identity Disorder - a Systematic Record of the Strategies to Reduce the Strain
}

\author{
Jelena Helmer ${ }^{1}$, Erich Kasten ${ }^{2}$ \\ ${ }^{1}$ Medical School Hamburg, University of Applied Sciences, Hamburg, Germany \\ ${ }^{2}$ Dept. of Neuropsychology, Medical School Hamburg, Hamburg, Germany
}

Email address:

je_lena@hotmail.de (J. Helmer), EriKasten@aol.com (E. Kasten)

\section{To cite this article:}

Jelena Helmer, Erich Kasten. Body Integrity Identity Disorder - a Systematic Record of the Strategies to Reduce the Strain. Psychology and Behavioral Sciences. Vol. 4, No. 4, 2015, pp. 139-146. doi: 10.11648/j.pbs.20150404.11

\begin{abstract}
People with Body Integrity Identity Disorder feel a discrepancy between their outward appearance and their sensed inner body image. To help these persons it is of major importance to find out how they deal with their disease in every day's life. What leads to a reduction of the wish for an amputation, paralysis or any other kind of disability? What strengthens the desire? Therefore this study deals with a systematic record of strategies and methods used by BIID affected persons for handling with their disease. Method: Hypotheses were formulated which concern the experience of different strategies, activities or situations with regard to the individual wish manifestation. For the investigation of the hypotheses as well as the record of the socio-demographic data a questionnaire with 42 items was created at an online platform specifically designed for scientific studies. Sample: 34 evaluable data sets could be collected. 24 persons $(70.6 \%)$ were male and 10 persons $(29.4 \%)$ were female. The average age amounted 40.26 years. 15 participants indicated to wish an amputation, eleven persons yearned for paraplegia, two concerned people indicated the wish to be bladder incontinent, one person yearned for blindness and one more indicated just the wish to sit in a wheelchair. Four participants did not make a statement to their individual wish manifestation. Results: Except the pretending-behavior, which is used by $47.1 \%$ of the participants to handle with the disease, could not be found a strategy, activity or situation which is sensed as wish reducing without exception by all participants. Summing up the collected results, they show a high individuality regarding the experience of wish reducing and wish increasing factors. On the other hand the results show that the perception of disabled people and any activity with BIID leads to a strengthening of the desire. Conclusion: Almost half of the participants are using the pretending-behavior by handling BIID. Pretending leads to a short-term reduction of the wish for amputation reasoned by appeared positive emotions. Long-termly this behavior though seems as an intensifier and holds up the disease and therefore the desire for amputation. Persons affected by BIID should try to extinguish personally experienced wish-increasing factors as well as to integrate wish-reducing strategies in many areas of their life as possible. Disease providing methods need to be identified and substituted with other techniques.
\end{abstract}

Keywords: Body Integrity Identity Disorder, BIID, Apotemnophilia, Amputee Identity Disorder, Xenomelia

\section{Introduction}

Body Integrity Identity Disorder (abbreviated: BIID) is in contrast to many other psychological diseases a very much unexplored disorder up to now. People with BIID describe the feeling that specific body parts do not belong to the own self $[8,9,22]$. Concerned persons cannot identify their selves with their intact body and strive for a condition that is being frightened by most of the people - they wish for a physical disability $[8,9,21,22]$. The desire for an amputation mostly concerns the left leg, although principally each and every body part can be affected $[2,6,9,12]$. People suffering from BIID are generally able to describe precisely where a wished amputation seam should pass. The localization is often reasoned pragmatically. For example would the loss of the right leg cause problems by driving a car so that the amputation of the left leg is preferred $[6,8]$. The wish for an amputation can change from one to another body side over the time $[8,11,12,22]$. Besides the desire for losing a specific limb there exist also wishes for other kinds of disabilities such as loosing senses or becoming paraplegic [9, 22]. BIID patients are aware of the absurdity of their desire. They know that their wish is not "normal", they are ashamed for their thoughts and therefore they often live alone with this deep desire $[8,9,22]$. The wish for disability is experienced as an 
imposing thought, which is present in most everyday life situation $[10,22]$. Some concerned people imitate the wished physical condition with a behavior called "pretending". Pretenders are tying high one leg or they are using crutches or a wheelchair for coming closer to the felt inner body image [3, $9,10,12,16,22]$.

Until now, several individual case studies are published, some group studies and interviews and only few experimental investigations. Besides these studies a few theoretical considerations and explanation approaches were published as well as articles that discuss ethical aspects [22]. The knowledge about this disease and the causes as well as successful treatment methods is limited. This proved a study of Neff and Kasten in 2009. The aim was to find out how many psychologists and psychotherapists know BIID in general. The participants were interrogated on basis of a realistic case study of a BIID affected person. 37.3\% categorized the disorder in the chapter F6 (Personality and behavior disorder). A considerably low proportion $(30.9 \%)$ indicated the diagnosis F4 (Neurotic, strain and somatoform disorder), $10.8 \%$ indicated the answer "I don't know." [17].

Primarily the disorder was called "Apotemnophilia", later "Amputee Identity Disorder". The today common term "Body Integrity Identity Disorder" goes back to the greatest executed group study "Desire for amputation of a limb: paraphilia, psychosis, or a new type of identity disorder" with 52 BIID affected people by Michael First [6]. This American psychiatrist recognized that BIID is an independent mentally disease that could be best compared with "Gender Identity Disorder". Furthermore he was interested in the motives for an amputation of the affected persons. About two third of the participants indicated as primary motive that an amputation would dissolve the discrepancy between the real outward body and the sensed inner body image. They could only reach the feeling of the completion of their identity by the loss of the body part, which is sensed as redundant. Besides the similarities which BIID shows to gender identity disorder, only few parallels to other already classified psychological diseases exist, e.g. body dismorphic disorder, mania operativa or the artificial syndrome. Despite commonalities, BIID could not be assigned clearly to one of these disorders so far. Nevertheless until now no criteria for a definition of BIID exist, so that the disease is not registered in any clinically relevant classification systems [22].

Typical for BIID is an early manifestation [3, 6, 8, 22]. By nearly all affected persons it happens between the age of four and twelve years, strengthens in the puberty and fortifies with increasing age $[6,8,22]$. Uncertainty consists concerning the frequency of the disorder. Striking is, that men are significantly more often affected than women. From 52 participants within the study of First were only four female [6]. Other researchers proved this conspicuity as well $[3,13,19$, 22]. The reasons for this fact are not known until today. Affected persons often show an above average education level, they mainly work in leading positions and mostly they are very successful and busy in every day's life $[6,22]$. Furthermore one can recognize a few overlaps in the personality structure of concerned persons. They are very purposefully, conscientious, self-confident, structurized and often show narcistic approaches. A continuous striving for challenges is also characteristically. The life as a disabled person is seen as a challenge as well [22]. The majority of BIID patients still have the wish for an operation although knowing the limitations and consequences that are connected with a disability $[11,13,22]$. It is a discussion topic for a lot of ethic-medical debates whether amputations of healthy extremities are acceptable. A few researchers, for example Müller, state against legal surgical amputations. According to this, Müller emphasizes the small research situation regarding possible treatment forms until now and she underlines the irreversibility of such extreme decisions [16]. In contrast, First as well as Bayne and Levy are holding the view that an amputation should be taken into consideration when other treatments had failed. They point out that affected people are more satisfied after achieving the desired condition and that the majority does not wish further operations $[1,3,6,8,16$, 20]. Noll \& Kasten confirmed this in a study. They investigated 19 already amputated BIID affected persons. None of the participants reported a deterioration of the general living situation. $78.9 \%$ could identify themselves with the own body much better after the operation and also the psychological condition changed positively [19].

There are different explanation approaches for BIID. To explain such an unknown and complex disorder a multi causal explanation model is required. In addition only a few therapy approaches exist, which could be helpful for the treatment of BIID. Kroeger et al. researched the success of different therapies in 25 BIID affected persons. 24\% participants indicated to have performed a cognitive behavioral therapy and $20 \%$ a psychodynamic therapy. For both groups the results emphasize that the wish for an amputation increased through the therapy on the one hand but that the physical strain was able to be reduced slightly on the other hand [13].

\section{Goal of This Study}

As already mentioned, the state of knowledge about BIID is limited. Until now no successful therapy concept is available which softens the wish for an amputation. Additively, there is no scientific founded definition of the disorder $[6,22]$. The few so far executed studies could only illustrate that most of the affected persons are not psychiatrically disturbed. Due to the lack of knowledge, the publicity often responds with incomprehension as well as denunciation towards the affected people. In shame and in fear of rejection the disease is often being kept as a secret, which in turn strengthens the physical strain. The disease leads to limitations in the personal, social and occupational life. A few patients even reported of suicide thoughts $[6,17,20,22]$.

It is unclear, how BIID affected persons can live for decades - most secretly - with their disease. There are unspecific individual advices of affected persons in internet-forums how to handle with the disease, but these are never recorded systematically. For some people it is helpful to perform 
pretending-behavior or to see images of people with disabilities. Others try to distract themselves from the obtruding thoughts.

The following study should clarify, how affected persons help themselves to control the pressure caused by the desire for a disability in every day's life. What is sensed as wish reducing? What strengthens the desire for a disability? The goal is to find out, whether there are certain strategies, activities or situations in every day's life, which are experienced as helpful, because they lead to a reduction of the wish. It is also of importance to find out what increases the wish. On basis of these findings further approaches of therapies could possibly be created so that BIID affected persons could be helped in future.

A strategy, which is inserted by many BIID affected people, is the pretending-behavior. It is unclear how many people do this at all and whether the imitation of the desired condition leads to a long-term reduction of the wish for disability. Similarly it is interesting how affected persons experience every day's activities, the own work or to be together with family or friends regarding the intensity of the own desire. Many people use music, journeys, sport, tasteful food or relaxing techniques for a compensation of the mostly stressful daily routine. Is of particular importance to find out whether BIID affected persons eventually experience those kinds of activities even as helpful by handling their disease. Based on these assumptions and the previous knowledge the following hypotheses were created: Persons try to achieve a reduction of the BIID-wish (for amputation, paralysis or any other kind of disability) due to: pretending behavior ( $\mathrm{H} 1)$, society of trusted people (H2), any kind of occupations (H3), work (H4), vacation (H5), pleasurable food (H6), sexual behavior (H7), sports (H8), relaxing techniques (H9), and music (H10). On the other hand, two hypotheses were formulated to investigate increasing factors for BIID: The perception of disabled people (H11), and any occupation with BIID leads to a strengthening of the BIID-wish (H12).

\section{Methods}

To be able to verify these hypotheses statistically a questionnaire with 42 items was created. The self-created questions were compiled with the internet platform "SosciSurvey", which is specifically designed for scientific surveys. The link to the questionnaire was published in the member zone of the "Forum for people with BIID and their relatives and friends in the German-speaking area" (http://www.forum.biid.ch). Only affected persons were interrogated. Additively to specific items, which recorded wish reducing and wish increasing factors, socio-demographic data as gender, birth date, education-level and the status of the relationship were collected. In a few open questions the participants were asked to describe the personal wish manifestation as well as to write a list of personal inserted strategies to handle with the disease. It was also questioned, whether the current (or previous) partner and the family are informed about the BIID-disease. An inner consistence of indicated answers could be secured by inserted reliability questions as well as calculated correlations between some items. Depending on the kind of the question three different alternatives for answers were given: (i) a dichotomic selection between don't agree and agree, (ii) a three stepped scale (don't agree, sometimes, agree), or (iii) an eleven-stepped scale from not applicable (1) to applicable (11). The answer alternatives selected by the participants were automatically saved by "SosciSurvey".

Using "SPSS", the averages and the standard deviations of single items as well as the frequency of the indicated answers and some correlations between different items were calculated. The Spearman's Rho-correlations coefficient was used and it was tested one-sided at a level of $\alpha=5 \%$.

\section{Sample}

The sample covered 34 BIID affected persons in total, whereof 24 persons $(70.6 \%)$ were male and 10 persons $(29.4 \%)$ were female. The average age amounted 40.26 years ( 24 to 55 years). The age of three participants could not be included due to missing answers. 19 persons specified to have studied. Among others, computer science $(n=3)$, medicine $(n=3)$ and biology $(n=2)$ were indicated. In 11 of the participants $(32.4 \%)$ the family knew about the existing disease. 22 persons $(64.7 \%)$ lived in a current relationship. In $50 \%(n=11)$ of these cases the current partner was informed about the existing disorder.

15 of the participants indicated the wish to be amputated. The goal mostly concerned the right leg, three persons liked to have both legs being amputated. Single participants indicated the left upper arm, the left hand and the right arm as unwanted extremities. One participant wished an amputation of the right arm and of the right leg. Eleven persons yearned for paraplegia. Nine of them made precise indications about wherefrom they liked to be paralyzed. In this regard, a paralysis starting at the thoracic spine was preferred $(n=6)$. In addition, two affected persons indicated the wish to be bladder incontinent. One person yearned for blindness and one indicated just the wish to sit in a wheelchair. Four participants made none ore non-evaluable indications regarding the individual desire. The distribution of the individual wish manifestation is shown in figure 1.

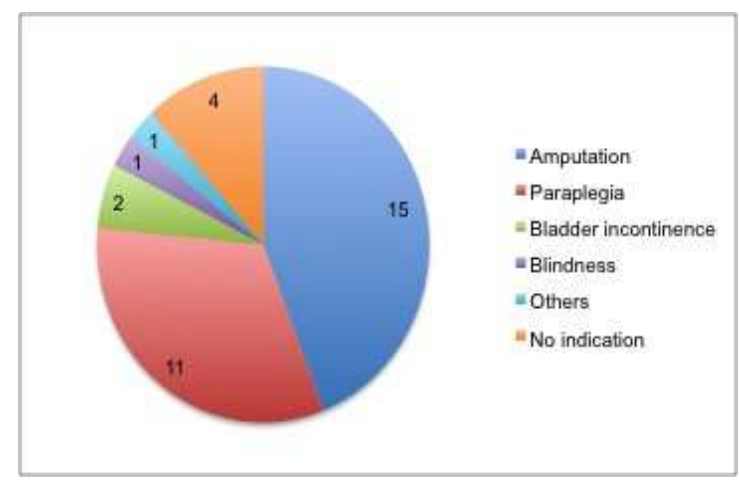

Figure 1. Distribution of the individual wish manifestation for the complete sample $(n=34)$. 


\section{Results}

After the record of socio-demographic data the participants were asked which strategies/methods were used to handle the wish for amputation, paralysis or any other kind of disability. Referring to this, five persons (14.7\%) indicated that only the establishment of the yearned condition (in most cases an amputation) could lead to a healing of the symptoms.

Hypothesis H1: The so-called pretending leads to a reduction of the wish for amputation, paralysis or any other kind of disability.

14 persons selected "don't agree", 10 persons "sometimes" and 6 persons "agree" with regard to the item "I pretend regularly (3-5 times a week) ". An average of $4.15 \pm 3.58$ on an eleven-stepped scale from not applicable to applicable was determined for the item "Pretending leads to a reduction of my wish".

Table 1. Frequency distribution of the indicated answers for the items that record the duration of the positive effect of the pretending-behavior by persons who selected "sometimes" or "agree" for the item "I pretend regularly (3-5 times a week)" ( $n=16)$.

\begin{tabular}{llll}
\hline & Don't agree & sometimes & agree \\
\hline The reduction of my wish lasts only during the pretending. & $6(37.5 \%)$ & $3(18,75 \%)$ & $7(43,75 \%)$ \\
The reduction of my wish lasts over several hours due to pretending. & $5(31,25 \%)$ & $6(37.5 \%)$ & $5(31,25 \%)$ \\
The reduction of my wish lasts over days due to pretending. & $9(56,25 \%)$ & $4(25 \%)$ & $3(18.75 \%)$ \\
\hline
\end{tabular}

A significant medium connection exists with $\rho=0.439$ at a level of $\alpha=5 \%$ between the items "I pretend regularly (3-5 times a week)" and "Pretending leads to a reduction of my wish". Moreover it was questioned on a three-stepped scale from "not applicable" over "sometimes" up to "applicable", whether an inhibition of the wish due to the pretending-behavior is experienced only in doing so, over hours or over days. Concerning this, table 1 shows the frequency distribution of the indicated answers of the persons who selected either "sometimes" or "agree" $(n=16)$ for the item "I pretend regularly (3-5 times a week)".

The item "I pretend regularly (3-5 times a week)" correlates significantly with an experienced positive effect during the pretending $(\rho=0.387)$, over hours $(\rho=0.626)$ and over days $(\rho=0.565)$. An average of $5.15 \pm 4.48$ was detected on an eleven-stepped scale for the item "I pretend in presence of trusted people". The item "I pretend regularly (3-5 times a week)" correlates equally significantly with the item "I pretend in present of trusted people". According to this Spearman's Rho amounts $\rho=0.609$. The items "My family knows about my wish" and the item "I pretend in presence of trusted people" also stands in a significant connection $(\rho=0.371)$. However the pretending in presence of trusted people does not correlate with the item "My partner knows about my wish". A low average of $3.12 \pm 3.14$ was detected on an eleven-stepped scale for the item "I avoid contact to others, so that I have enough time to pretend".

$\mathrm{H} 2$ : The society of trusted people leads to a reduction of the wish for amputation, paralysis or any other kind of disability.

The average of the item "My wish is less pronounced in society of trusted people" amounts $4.12 \pm 3.27$ on an eleven-stepped scale. The endpoint "applicable" was selected by none of the questioned persons. A relatively low average of $3.53 \pm 3.04$ on an eleven-stepped scale was detected for the item "Conversations with trusted people lead to a reduction of my wish". A significant medium connection $(\rho=0.384)$ exists at a level of $\alpha=5 \%$ between the items "My wish is less pronounced in society of trusted people" and "Conversations with trusted people lead to a reduction of my wish". With 8.21 \pm 3.39 a high average was recorded on an eleven-stepped for the item "My wish is strong pronounced if I am alone".
However $44.1 \% \quad(n=15)$ of the participants denied the statement "I often do not like to be alone, because then my wish strengthens" with "not applicable". Only $11.8 \%(n=4)$ selected the endpoint "applicable". Regarding to this, the average is $3.88 \pm 3.44$ on an eleven-stepped scale. Moreover, it was questioned whether the participants try as frequently as possible to meet with trusted persons. Here, the average is $6.24 \pm 3.64$ on an eleven-stepped scale. A significant connection at a level of $\alpha=5 \%$ with $\rho=0.375$ could be found between the items "I often do not like to be alone, because then my wish strengthens" and "I try to meet with trusted people as frequently as possible", which emphasizes that the items were answered with sufficient reliability.

H3: Daily occupations lead to a reduction of the wish for amputation, paralysis or any other kind of disability.

The average of the item "My wish is strongly pronounced, if I follow daily occupations" amounts $8.06 \pm 2.81$ on an eleven-stepped scale. An average of $7.68 \pm 3.39$ was calculated for the item "Daily occupation does not lead to a reduction of my wish".

H4: My own work leads to a reduction of the wish for amputation, paralysis or any other kind of disability.

The average for the item "My wish is less strongly present during work" is on an eleven-stepped scale located at 5.12 \pm 3.49 . A similar result with an average of $5.21 \pm 3.71$ was calculated regarding the item "My work has a positive effect on the reduction of my wish". Spearman's Rho of the just mentioned items amounts $\rho=0.593$ and is at a level of $\alpha=5 \%$ significant. This result underlines once again the inner consistence of the indicated answers.

H5: Vacation leads to a reduction of the wish for amputation, paralysis or any other kind of disability.

The average of the item "My wish is less pronounced in a vacation compared with being at home in every day's life" amounts $3.68 \pm 3.43$ on an eleven-stepped scale. It was also questioned, whether vacation leads to a strengthening of the personal desire. According to this, the average amounts 5.26 \pm 3.61 on an eleven-stepped scale. It get's clear that more persons experience a strengthening than a reduction of the wish reasoned by vacation.

H6: A pleasurable food leads to a reduction of the wish for 
amputation, paralysis or any other kind of disability.

The average of the item "I think less frequently about my wish during a pleasurable food" amounts $4.65 \pm 3.961$ on an eleven-stepped scale. Furthermore, it was questioned on a three-stepped scale from "don't agree" to "sometimes" up to "agree", whether a pleasurable food leads to a reduction of the wish for more hours. "Don't agree" was indicated by $64.7 \%$ $(\mathrm{n}=22)$, "sometimes" by $23.5 \%(\mathrm{n}=8)$ and "agree" by $11.8 \%$ $(n=4)$ of the participants.

H7: Sex leads to a reduction of the wish for amputation, paralysis or any other kind of disability.

The item "Sex helps to reduce my wish short-termly" was answered by $41.2 \%(\mathrm{n}=14)$ with "don't agree", by $41.2 \%$ $(n=14)$ with "sometimes" and by $17.6 \%(n=6)$ with "agree". Thus, $41.2 \%(\mathrm{n}=14)$ of the participants experience sometimes, $17.6 \%(n=6)$ more often a short-term reduction of the wish by having sex. Likewise it was questioned, whether the wish for amputation is always present during sex. According to this, the average amounts $6.47 \pm 3.510$ on an eleven-stepped scale. A significant negative connection between the items "Sex helps to reduce my wish short-termly" and "My partner knows about my wish" could be calculated $(\rho=-0.365)$.

H8: Sports leads to a reduction of the wish for amputation, paralysis or any other kind of disability.

An average of $3.85 \pm 3.33$ on an eleven-stepped scale was calculated for the item "My wish is less pronounced if I do sports". The average of the item "I don't do sports, because then my wish strengthens" amounts $3.09 \pm 3.11$ on an eleven-stepped scale.

H9: Relaxing techniques lead to a reduction of the wish for amputation, paralysis or any other kind of disability.

It was interrogated, whether relaxing techniques are being inserted by handling the own disease. With regard to this, the average amounts $2.71 \pm 2.99$ on an eleven-stepped scale. $61.8 \%$ $(n=21)$ of the sample decided for the endpoint "not applicable", $5.9 \%(n=2)$ selected the endpoint "applicable". The average of the item "Relaxing techniques lead to a reduction of my wish" is located at $4.56 \pm 3.457$ on an eleven-stepped scale.

H10: Music leads to a reduction of the wish for amputation, paralysis or any other kind of disability.

The average of the item "Music helps to reduce my wish for a few moments" amounts $5.00 \pm 3.38$ on an eleven-stepped scale from "not applicable" to "applicable".

H11: The perception of disabled persons strengthens the own wish for amputation, paralysis or any other kind of disability.

A very high average of $9.15 \pm 2.94$ on an eleven stepped scale was determined for the item ,If I see people, who have my desired disability, my own wish strengthens". In addition to that a high average of $8.15 \pm 3.50$ was also found for the item "I am jealous if I see people who have the disability I wish for myself". With $\rho=0.469$ at a level of $\alpha=5 \%$ there is a significant strong connection between these items. It was also interrogated, whether the perception of disabled persons leads to a reduction of the own desire. With regard to this, the average amounts $3.26 \pm 3.40$ on an eleven-stepped scale from "not applicable" to "applicable". The sensed reduction of the wish due to the view of disabled persons correlates significantly negative with the item "I am jealous if I see people, who have the disability that I wish for myself" $(\rho=-0.420)$.

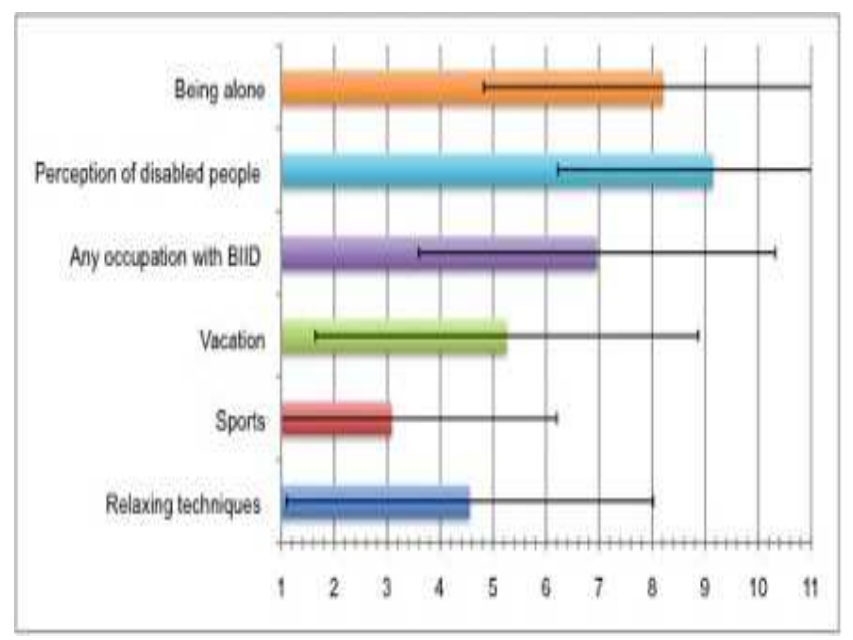

Figure 2. Results of the items, that measured a strengthening of the wish for disability based on different activities on an eleven-stepped scale (not applicable (1) to applicable (11)).

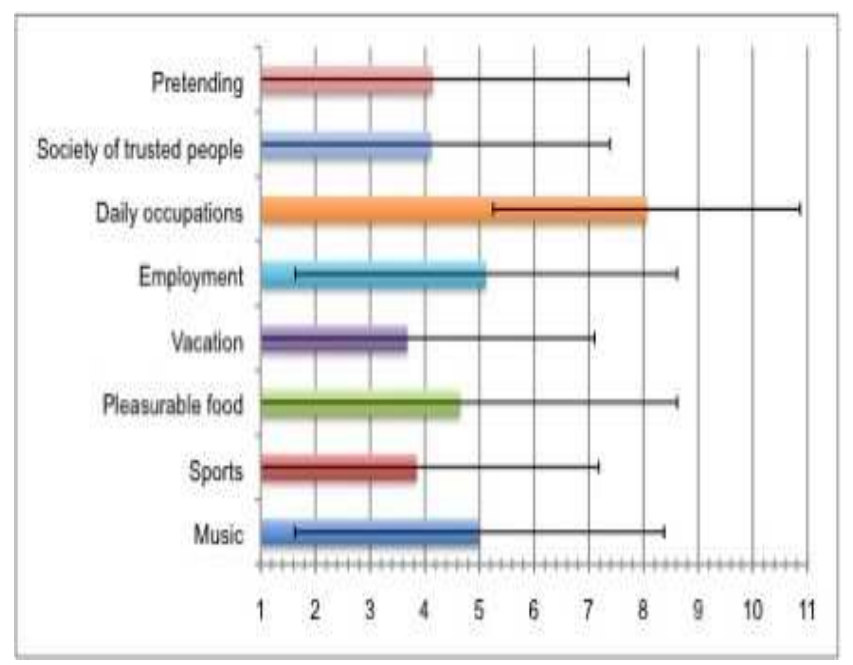

Figure 3. Results of the items, that measured a reduction of the wish for disability based on different activities on an eleven-stepped scale (not applicable (1) to applicable (11)).

H12: Each occupation with BIID leads to a strengthening of the wish for amputation, paralysis or any kind of disability.

The average of the item "Each occupation with my wish for disability strengthens the desire" amounts $6.97 \pm 3.36$ on an eleven-stepped scale. This result is underlined with an average of $7.50 \pm 3.17$ for the item "The more I occupy myself with my wish, the stronger it gets". Moreover, it was questioned on a three-stepped scale, whether distraction results in a reduction of the wish for amputation, paralysis or any other kind of disability. "Don't agree" was selected by $29.4 \% \quad(n=10)$, "sometimes" by $58.8 \%(n=20)$ and "agree" by $11.8 \%(n=4)$ of the sample. There is a significant connection of $\rho=0.297$ at a level of $\alpha=5 \%$ between the items "Each occupation with my wish for disability strengthens this" and "Each distraction 
leads to a short-term reduction of my wish", which once again shows that the questions were answered with sufficient reliability.

\section{Conclusion}

BIID-affected persons, who perform strategies to handle with their disease, most frequently use the method of pretending. Therefore, it is of outmost importance to lighten up this behavior. In total, $47.1 \%(n=16)$ of the participants indicated to show pretending-behavior sometimes or regularly. The usual frequency was three to five times a week. Regular pretenders experience a significant reduction of the wish for a disability due to this behavior. The here found correlation explains why the pretending-behavior is being inserted again and again at all. The duration of the experienced reduction of the desire due to pretending is individually different; this seems to depend on the intensity and the individual type of the pretending-behavior. Persons, who imitate the desired condition regularly, often show the behavior even in the presence of trusted people. In some other cases the pretending-behavior leads to an avoiding of social contacts. This fact is probably associated with shame and fear experienced by many BIID affected persons and the resulting secret-keeping of the disease.

In summary, pretending helps the BIID affected persons for some time, but the cognitive-behavioral perspective evaluates the imitation of a strived disabled condition as problematic. It is generally understandable that a strategy, which leads to a short-term reduction of the wish for amputation, is performed over and over again. Pretending is experienced as pleasurable and is associated with a rising of the welfare and the general mood as well as an extraordinarily recognition of the environment. But long-termly it appears as a strengthener due to the sensed positive emotions and maintains the disease and therefore the wish for amputation. Probably only a few affected persons know the maintaining character of the pretending-behavior and try to "suppress" this knowledge. With regard to this, it is of outmost importance to do explanatory work and to underline the long-termly negative effect of this strategy. The primary goal should be to find adequate and relieving alternatives.

The results show furthermore, that the wish is strengthened in many cases if the affected persons are alone. However the majority of the sample does not try to avoid being alone, because a partner or other people interfere with the carrying out of the pretending behavior. On the other hand, many participants try as often as possible to meet trusted people. This aspect stands in a connection with efforts to avoid being alone. In general, the being-together with trusted people does not seem to reduce the wish in general. This is also valid for conversations with trusted people. Between the items "My wish is less pronounced while being in society of trusted people" and "Conversations with trusted people lead to a reduction of my wish" a significant correlation was found. The secret-keeping of the disease surely plays a huge role hereby. Affected persons, who have informed their personal surrounding about their disease, experience society and conversations probably more positive with regard to the own wish than persons who try to keep their disease continuously as a secret.

The perception of disabled people leads to a strengthening of the wish for amputation by the majority of the participants. Connected herewith, many persons even experience the feeling of jealousy, which surely is achieved partly out of the knowledge about the non-realization of the own desire. Only a few participants indicated that the sight of disabled persons leads to a reduction of the wish. The results show furthermore, that each occupation with the BIID-desire leads to a strengthening of the wish in many cases. Because of that, distraction is often experienced as wish reducing. This result is possibly based on the extreme aim for performance, which is often visible in BIID affected persons as well as the continuous searching for challenges. Managing every day's life with a disability can prove own abilities.

A few participants indicated, that the wish is less pronounced during work. Whether the own work is evaluated positively with regard to a reduction of the wish is surely influenced by the type of the employment and the individual meaning of the own work.

Surprisingly the results show a strengthening of the wish by affected persons during vacation. As an explanation it could be supposed that in the holidays the person has more time to ruminate about the pros and cons of an amputation.

More than half of the participants admitted a short-term reduction of the wish due to sexual intercourse. On the one hand, sexual behavior supports a short-term distraction in which the wish for disability moves into the background. A few participants indicated in an open question, that even masturbation and introduction of the desired disability into the sexual life is helpful to handle with the disorder. As far as the partner allows, sex on the other hand probably offers the possibility to imitate the desired condition and therefore to live the own wish for disability in a very intensive and intimate situation. Furthermore, many participants indicated, that the wish is continuously present during sex. Surely, the thereby existing intensive body reference plays a decisive role. For following researches it would be extremely interesting, to investigate the experienced short-term reduction of the wish caused by sex as well as the presence during it in dependence of the sexual components, which could be found in conjunction with Body Integrity Identity Disorder.

Doing sports has positive health effects for many people in general, in addition it distracts from negative thoughts and feelings. This is also valid for a few BIID affected persons. On the other hand, a few participants indicated that sports leads to a strengthening of the wish though, because it focuses the attention on the body and thus also on the part of the body to which the amputation-request relates. Relaxing techniques are also often experienced as wish strengthening. These results are likewise based on the strong body reference doing these activities. The thoughts are directed to the body, with which the affected persons cannot identify themselves 
so that the wish for disability is being promoted. Nevertheless, a few participants indicated that they insert relaxing techniques to handle with their disease. Surely the type of the technique is of outmost importance hereby.

Music is experienced as wish reducing by a few participants; others do not experience a reduction. This result probably depends on the individual relevancy of music.

The results show, that not only the BIID-Disorder itself is extremely complex and heterogeneous. Also the personal handling and the experienced wish reducing or strengthening factors are very individual. It is important to support affected persons by finding personally experienced wish reducing factors and to find a way to integrate these in many areas of life (as far as they do not illustrate a problem behavior). These individually experienced relieving strategies have to be interrogated by a therapist. Disease encouraging methods need to be recognized and replaced by wish-reducing alternatives. However, this is only possible if BIID affected persons allow this and participate actively.

In personal correspondence with BIID affected persons it became obvious that the existing strain does not result just only out of the wish itself. Furthermore the uncertainty with regard to causes, the limited state of knowledge, the far-reaching anonymousness of the disease in the society as well as the knowledge of the non-feasibility of the whished physical state are burdensome factors. Only in about one third of the participants the family knew about the existing disease. This emphasizes the necessity to afford explanatory work with the aim to inform the publicity appropriately and precisely about the disease, so that the affected persons do not have to suffer additionally by a strong "secret-keeping pressure". BIID is a serious disorder and it cannot be dismissed as an absurd perversion. The individual needs of BIID affected persons differ strongly from general valid social conventions. In spite of their healthy limbs BIID-affected persons experience themselves as ill and they want to be healthy due to an amputation. This desire collides massively with the social perception of health in general. The imagination to stumble a healthy and functioning body and therefore making a healthy person "ill" is barely comprehensible for non-affected persons. Thus, one reacts with a lack of understanding, distraction, rejection and condemnation at affected persons. Especially in the course of inclusion, these inappropriate reactions have to be counteracted. The inhibition thresholds of affected persons to entrust their personal surroundings and to search for help could be reduced due to the ending of such a taboo in the society. Just when people suffering from BIID ask for professional help, different therapy-approaches can be researched, modified and possibly finally lead to a success in the future.

The individual preferences of methods to handle with the disease may be based on different types of BIID. The number of affected people is too small for a cluster analysis of sub-types. However such differences could affect the choice and the efficacy of strategies. In following researches the intrinsic/extrinsic motivation should be controlled.

\section{Acknowledgements}

The authors thank all participants of this study.

\section{References}

[1] Bayne, T., Levy, N. (2005). Amputees By Choice: Body Integrity Identity Disorder and the Ethics of Amputation. Journal of Applied Philosophy, 22(1), 75-86.

[2] Blanke, O., Morgenthaler, F. D., Brugger, P., Overney, L. S. (2009). Preliminary evidence for a fronto-parietal dysfunction in able-bodied participants with a desire for limp amputation. Journal of Neuropsychology, 3, 181-200.

[3] Blom, R., Hennekam, R., Denys, D. (2012). Body Integrity Identity Disorder. PLoS ONE, 7(4), e34702. doi:10.1371/journal.pone.0034702.

[4] Bortz, J. (2010). Statistik für Human- und Sozialwissenschaftler. Berlin Heidelberg: Springer-Verlag.

[5] Bruno, R. (1997). Devotees, Pretenders and Wannabes: Two Cases of Factitious Disability Disorder. Journal of Sexuality and Disability, 15(4), 243-260.

[6] First, M. B. (2004). Desire for amputation of a limb: paraphilia, psychosis, or a new type of identity disorder. Psychological Medicine, 34, 1-10.

[7] Furth, G.M, Smith, R. (2000). Amputee Identity Disorder: Information, Questions, Answers and Recommendations About Self-Demand Amputation. AuthorHouse Paperback.

[8] Kasten, E. (2009). Body Integrity Identity Disorder (BIID): Befragung von Betroffenen und Erklärungsansätze. Fortschritte Neurologie Psychiatrie, 77, 16-24.

[9] Kasten, E. (2012). Body Integrity Identity Disorder Körperidentität durch erwünschte Behinderung. Psychiatrie und Psychotherapie up2date, 6(3), 165-177.

[10] Kasten, E. (2013). I feel like I was in the wrong body. AlJazeera (online magazine). http://www.aljazeera.com

[11] Kasten, E., Spithaler F. (2009). Body Integrity Identity Disorder: Personality Profiles and Investigation of Motives. In: Stirn A, Thiel A, Oddo E, editors. Body Integrity Identity Disorder. Pabst Science Pubishers, 20-40.

[12] Kasten, E., Stirn, A. (2009). Body Integrity Identity Disorder (BIID). Wechselnder Amputationswunsch vom linken auf das rechte Bein. Zeitschrift für Psychiatrie, Psychologie und Psychotherapie, 57(1), 55-61.

[13] Kroeger, K., Schnell, T. Kasten, E.: Effects of psychotherapy on patients suffering from Body Integrity Identity Disorder. American Journal of Applied Psychology. 2014; 3(5): 110-115

[14] McGeoch P., Brang D., Song T., Lee R., Huang M., et al. (2011). Xenomelia: a new right parietal lobe syndrome. Journal of Neurology, Neurosurgery \& Psychiatry, 82, 1314-1319.

[15] Money J, Jobaris R, Furth G. (1977). Apotemnophilia: two cases of self-demand amputation as a paraphilia. Journal of Sex Research, 13(2), 115-125.

[16] Müller, S. (2007): Dieses Bein will ich nicht. Gehirn und Geist, Juli/August, 66 . 
[17] Müller, S. (2009): Body Integrity Identity Disorder (BIID) - Is the Amputation of Healthy Limps Ethically Justified?. The American Journal of Bioethics, 9, 36-43.

[18] Neff, D. \& Kasten, E. (2009). BIID: What do health care professionals know? European Journal of Counselling Psychology, 1(2), 16-30.

[19] Noll, S. \& Kasten, E. (2014). Body integrity identity disorder (BIID): How satisfied are successful wannabes? Psychology and Behavioral Sciences, 3(6), 222-232

[20] Ryan C, (2009). Amputating Healthy Limbs - Should peaople with body integrity identity disorder be allowed to amputate a healthy limb? Issues Magazine, 86, 31-33.

[21] Sedda, A., Bottini, G. (2014). Apotemnophilia, body integrity identity disorder or xenomelia? Psychiatric and neurologic etiologies face each other. Neuropsychiatric Disease and Treatment, 10, 1255-1265.
[22] Stirn, A., Thiel, A. \& Oddo, S. (2010). Body Integrity Identity Disorder (BIID) - Störungsbild, Diagnostik, Therapieansätze. Basel: Beltz Verlag.

[23] Forum for people suffering from BIID and their relatives and friends (Forum für Menschen mit BIID und deren Angehörige und Freunde im deutschsprachigen Raum): http://forum.biid.ch, 29.6.2014.

[24] Informations for BIID-sufferers, relatives, physicians and therapists (Informationsseite für BIID-Betroffene, Angehörige, Ärzte und Therapeuten): http://www.biid-dach.org, 15.07.2014

[25] Association for the promotion of studies of body identity disorders (Verein zur Förderung von Studien über Körperidentitätsstörungen): http://www.vfsk.info, 28.07.2014.

[26] Personal sources: Email-Correspondence with BIID affected people. 